\title{
ISOMETRIES OF COMPACT SUBMANIFOLDS OF A RIEMANNIAN MANIFOLD
}

\author{
CHUAN-CHIH HSIUNG \& BURGESS HAROLD RHODES
}

\begin{abstract}
Introduction
Let $M$ be a differentiable manifold of dimension $n \geq 2, x, x^{*}: M \rightarrow R$ two immersed compact submanifolds in a Riemannian manifold of dimension $n+m(m>0)$ admitting a special infinitesimal nonisometric conformal transformation $\xi$, and $f: x(M) \rightarrow x^{*}(M)$ a volume-preserving diffeomorphism. Conditions are found for (i) $f$ to be an isometry and (ii) hypersurfaces $x(M)$, $x^{*}(M)$ to have the same second fundamental form when $m=1$. In particular, when $R$ is a Euclidean space $E$, these conditions were obtained by Chern and Hsiung [1] for case (i), and by Hsiung [3] for case (ii), as in these cases, in $E$ the position vector of a point with respect to a fixed point 0 generates such a special infinitesimal nonisometric homothetic transformation.

In order to simplify the presentation of our work, as before we shall consider tensor products of multivectors and exterior differential forms. Differentiation of multivectors will be taken in the sense of equation (2.22), and differentiation of exterior differential forms will be exterior differentiation; multiplication of matrices will be by the usual row-by-column law.
\end{abstract}

\section{Lemmas}

Let $V$ be a real vector space of dimension $n(\geq 2)$, and let $G$ and $H$ be two bilinear real-valued functions over $V \times V$ so that $G$ and $H$ are completely determined by the values $g_{i k}=G\left(e_{i}, e_{k}\right)$ and $h_{i k}=H\left(e_{i}, e_{k}\right), 1 \leq i, k \leq n$, where the vectors $e_{1}, \cdots, e_{n}$ constitute a basis for the space $V$. Under a change of basis

$$
e_{i} \rightarrow e_{i}^{*}=t_{i}^{k} e_{k},
$$

the matrices $\left\|g_{i k}\right\|$ and $\left\|h_{i k}\right\|$ are changed to $T\left\|g_{i k}\right\|^{t} T$ and $T\left\|h_{i k}\right\|^{t} T$ respectively, where the repeated index $k$ is summed over its range, $T=\left\|t_{i}^{k}\right\|$, and ${ }^{t} T$ is the transpose of $T$. Consider the determinant

Communicated June 20, 1967. The work of the first author was partially supported by the National Science Foundation grant GP-4222. 


$$
\left|g_{i k}+\lambda h_{i k}\right|=\left|g_{i k}\right|+n \lambda P\left(g_{i k}, h_{i k}\right)+\cdots+\lambda^{n}\left|h_{i k}\right| \text {. }
$$

Since $\left|g_{i k}+\lambda h_{i k}\right|$ will be multiplied by $|T|^{2}$. under the change (1.1) of basis, the ratio of any two coefficients in the polynomial in $\lambda$ on the right side of equation (1.2) is independent of the choice of the basis $e_{1} \cdots e_{n}$ for $V$. In particular, if $G$ is nonsingular, that is, if $\left|g_{i k}\right| \neq 0$, the quotient

$$
H_{G}=P\left(g_{i k}, h_{i k}\right) /\left|g_{i k}\right|
$$

depends only on $G$ and $H$. For example, if $g_{i k}=\delta_{i k}(=1$ for $i=k$; $=0$ for $i \neq k)$, then $H_{G}=\sum_{i} h_{i i} / n$.

Since the construction of $H_{G}$ is linear in $H$, it can be generalized to a bilinear function $H$ over $V \times V$ with values in a vector space $W$, and $H_{G}$ is then an element in $W . H_{G}$ can be called the contraction of $H$ relative to $G$, or, in the language of tensors, $H_{G}$ is a vector in $W$ constructed from a covariant tensor $H$ of order two with values in $W$ relative to a real-valued nonsingular covariant tensor $G$ of order two.

The following two lemmas will be needed in the proof of our main theorems.

Lemma 1.1. Let $V$ be a real vector space of dimension $n(\geq 2)$, and $G$ and $H$ symmetric positive definite bilinear real-valued functions over $V \times V$ completely determined by the values $g_{i k}=G\left(e_{i}, e_{k}\right)$ and $h_{i k}=H\left(e_{i}, e_{k}\right)$, $1 \leq i, k \leq n$, where $e_{1}, \cdots, e_{n}$ form a basis of the vector space $V$. Denote

$$
g=\left|g_{i k}\right|, \quad h=\left|h_{i k}\right| .
$$

Then

$$
H_{G} \geq(h / g)^{1 / n},
$$

where the equality holds when and only when $h_{i k}=c g_{i k}$ for a certain $c$.

The inequality (1.5) is one in a class of inequalities for hyperbolic polynomials given by Gårding [2]. A direct and elementary proof of the inequality (1.5) was given by Rhodes [4] by making use of the generalized arithmeticmean-geometric-mean inequality. A still simpler proof of the inequality (1.5) is obtained as follows: Since both matrices $G$ and $H$ are positive definite, we can choose the matrix $T$ of a change of basis such that $G$ be the unit matrix and $H$ a diagonal matrix. Then by the generalized arithmetic-mean-geometricmean inequality we obtain

$$
H_{G}=\frac{1}{n} \sum_{i} h_{i i} \geq\left(h_{11} \cdots h_{n n}\right)^{1 / n}=(h / g)^{1 / n},
$$

where the equality holds when and only when $h_{i k}=c g_{i k}$ for a certain $c$.

Lemma 1.2. Let $e_{1}, \cdots, e_{n}$ be any $n$ vectors in a vector space $V$ of dimen- 
sion $n$, and $\xi_{1}, \cdots, \xi_{n}, \eta_{i}^{k}(i, k=1, \cdots, n)$ any elements of the dual space $V^{*}$ of $V$ such that

$$
\sum_{i} \eta_{i}^{i}=0
$$

Denote

$$
\begin{aligned}
& e={ }^{t}\left\|e_{1}, \cdots, e_{n}\right\|, \quad \xi=\left\|\xi_{1}, \cdots, \xi_{n}\right\|, \\
& w=\xi e, \quad \eta=\left\|\eta_{i}^{k}\right\|, \quad 1 \leq i, k \leq n .
\end{aligned}
$$

Then

$$
\eta e w^{n-1}-(n-1) e \xi \eta e w^{n-2}=0,
$$

where the product of elements in each space is the exterior product.

This lemma is due to Chern and Hsiung; for its proof see [1, p. 283].

\section{Submanifolds of a Riemannian manifold}

Throughout this paper the ranges of indices are given as follows, unless stated otherwise:

$$
\begin{gathered}
1 \leq i, j, k, \cdots \leq n, \\
1 \leq \alpha, \beta, \gamma, \cdots \leq n+m, \\
1 \leq r, s, \cdots \leq m(m>0) .
\end{gathered}
$$

Let $R$ be a $C^{2}$-Riemannian manifold of dimension $n+m$ with fundamental tensor $G$, and $R_{x}, R_{x}^{*}$ respectively the tangent space and its dual space at a point $x \in R$. By taking $C^{2}$-bases $e_{1} \cdots e_{n+m}$ and $\omega^{1} \cdots \omega^{n \div m}$ in the tangent spaces and their dual spaces over a neighborhood $U$ of the point $x \in R$, we then have

$$
\begin{array}{r}
d x=\omega^{\alpha} e_{\alpha}, \\
d e_{\alpha}=\omega_{\alpha}^{\beta} e_{\beta},
\end{array}
$$

where $d$ denotes exterior differentiation, the repeated indices imply summation, and the $\omega_{\alpha}^{\beta}$ are linear differential forms. Since $\omega^{1}, \cdots, \omega^{n+m}$ span the space of linear differential forms over the neighborhood $U$, we can write

$$
\omega_{\beta}^{\alpha}=\gamma_{\beta \sigma}^{\alpha} \omega^{\sigma},
$$

where $\gamma_{\beta \sigma}^{\alpha}$ are the components of the affine connexion $C$ of the Riemannian metric $G$ with respect to the frame $x e_{1} \cdots e_{n+m}$. 
Let $x^{1}, \cdots, x^{n+m}$ be a system of local coordinates in the neighborhood $U$ of the point $x$. Then

$$
d x^{\beta}=\omega^{\alpha} e_{\alpha}^{\beta},
$$

where $e_{\alpha}^{\beta}(\beta=1, \cdots, n+m)$ are the components of $e_{\alpha}$. Put

$$
\omega_{\beta}^{\alpha}=\Gamma_{\beta \sigma}^{\alpha} d x^{\sigma},
$$

where $\Gamma_{\beta \sigma}^{\alpha}$ are the components of the affine connexion $C$ in the local coordinates $x^{1}, \cdots, x^{n+m}$. Substituting equation (2.5) in equation (2.6), and comparing the resulting equation with equation (2.4), we then find

$$
\gamma_{\beta \sigma}^{\alpha}=\Gamma_{\beta \rho}^{\alpha} \epsilon_{o}^{\rho} .
$$

Let $M$ be an abstract manifold of dimension $n$, and consider an immersion $x: M \rightarrow R$, that is, a $C^{2}$-mapping $x$ of $M$ into $R$ such that the induced linear mapping $x_{*}$ on the tangent spaces is univalent everywhere. By choosing $e_{1}, \cdots, e_{n}$ to be in the tangent space of the manifold $x(M)$ at the point $x(p)$, $p \in M$, and denoting by the same symbols the forms on this submanifold of frames $e_{1} \cdots e_{n}$ induced by the mapping $x$, at the point $x(p)$ of the manifold $\boldsymbol{x}(M)$ we have, from equation (2.2),

$$
\omega^{n+s}=0 \quad(s=1, \cdots, m),
$$

and therefore

$$
\begin{aligned}
d x & =\omega^{i} e_{i}, \\
d e_{i} & =\omega_{i}^{\beta} e_{\beta} .
\end{aligned}
$$

Let $\xi$ be a vector field on the manifold $R$, so that

$$
\xi=\xi^{\alpha} e_{\alpha} .
$$

By using equations (2.3), (2.4), (2.8) we then obtain

$$
d \xi=\left(\nabla_{i} \xi^{\alpha}\right) \omega^{i} e_{\alpha},
$$

where $\nabla_{i}$ denotes the covariant derivative in the direction $e_{i}$, and $\nabla_{i} \xi^{\alpha}$ is given by

$$
\nabla_{i} \xi^{\alpha}=\xi^{\alpha}{ }_{i}+\xi^{\beta} \gamma_{\beta i}^{\alpha},
$$

$\xi^{\alpha}{ }_{i}$ being defined by

$$
d \xi^{\alpha}=\xi^{\alpha}{ }_{i} \omega^{i} .
$$


More generally, for a mixed tensor $\xi_{\beta}^{\alpha}$ we have

$$
\nabla_{i} \xi_{\beta}^{\alpha}=\xi_{\beta, i}^{\alpha}+\xi_{\beta}^{\alpha} \gamma_{o i}^{\alpha}-\xi_{\theta}^{\alpha} \gamma_{\beta i}^{\alpha} \text {. }
$$

Since

$$
d \xi^{\alpha}=\frac{\partial \xi^{\alpha}}{\partial x^{i}} d x^{i}
$$

substituting equation (2.5) in equation (2.16), and comparing the resulting equation with (2.14), we obtain

$$
\xi^{\alpha}, i=\frac{\partial \xi^{\alpha}}{\partial x^{k}} e_{i}^{k}
$$

which holds for any tensor. From equations (2.7), (2.17) it follows that if $e_{1} \cdots e_{n}$ is a natural frame, then $\nabla$ becomes the covariant differentiation in local coordinates. Thus, by the definition of tensors,

$$
\nabla_{i} g_{\alpha \beta}=0 \text {, }
$$

where $g_{\alpha \beta}$ are the components of the fundamental tensor $G$ of the manifold $R$. Moreover, on $R$ if $\xi$ generates an infinitesimal conformal transformation, then,

$$
\nabla_{\alpha} \xi_{\beta}+\nabla_{\beta} \xi_{\alpha}=2 \rho g_{\alpha \beta},
$$

where $\rho$ is a real-valued function on $R$. In particular, the infinitesimal conformal transformation generated by the vector field $\xi$ is homothetic or isometric according as $\rho$ is a constant function or $\rho \equiv 0$. On $R, \xi$ is said to generate an enlarging infinitesimal conformal transformation, if (2.19) holds with positive $\rho$. From equation (2.19) it follows that $\rho=\frac{1}{n+m} \nabla_{\alpha} \xi^{\alpha}$, and therefore, by means of the well-known theorem of Green, that if $R$ is compact and orientable, then $\int_{R} \rho d V=0$, where $d V$ is the volume element of the manifold $R$. Hence if $R$ is compact and orientable, then it does not admit any enlarging infinitesimal conformal transformations. We are indebted to $\mathrm{K}$. Yano for calling our attention to this point.

Now choose $e_{n+1} \cdots e_{n+m}$ to be an orthonormal frame in the normal bundle of $x(M)$. By introducing the matrices

$$
\begin{gathered}
\omega=\left\|\omega^{1}, \cdots, \omega^{n}\right\|, \\
e=\left\|\begin{array}{c}
e_{1} \\
\vdots \\
e_{n}
\end{array}\right\|, \quad a=\left\|\begin{array}{c}
e_{n+1} \\
\vdots \\
e_{n+m}
\end{array}\right\|
\end{gathered}
$$


equations (2.9), (2.10) then become

$$
d x=\omega e, \quad d e=\Omega e+\theta a,
$$

where

$$
\Omega=\left\|\omega_{i}^{k}\right\| \quad \theta=\left\|\omega_{i}^{n+s}\right\| .
$$

Exterior differentiation of the first equation of (2.22) gives

$$
d \omega=\omega \wedge \Omega, \quad \omega \wedge \theta=0 .
$$

The second equation of (2.24) can be written explicitly as

$$
\omega^{i} \wedge \omega_{i}^{n+s}=0 \text {. }
$$

By applying a well-known lemma of E. Cartan, from equation (2.25) we then have

$$
\omega_{i}^{n+s}=A_{i j}^{n+s} \omega^{j}, \quad A_{i j}^{n+s}=A_{j i}^{n+s} .
$$

These functions $A_{i j}^{n+s}$ defined on the submanifold $x(M)$ are coefficients in the second fundamental form $\Pi$ of $x(M)$. In fact, from equations (2.21) and (2.26) we obtain the second differential of $x$ in the ordinary sense:

$$
d^{2} \boldsymbol{x}=\left(d \omega^{i}+\omega^{j} \omega_{j}^{i}\right) \boldsymbol{e}_{i}+A_{i j}^{n+s} \omega^{i} \omega^{j} e_{n+s} .
$$

If $\nu$ is any normal vector of $x(M)$ at $x(p)$, then the second fundamental form relative to $\nu$ is given by

$$
\Pi_{\nu}=\left\langle\nu, d^{2} x\right\rangle=\left\langle\nu, e_{n+s}\right\rangle A_{i j}^{n+s} \omega^{i} \omega^{j},
$$

where $\langle$,$\rangle denotes the scalar product in the tangent space of the manifold$ $R$, and the multiplication of linear differential forms is commutative in the sense of the symmetric algebra.

For each normal field $\nu$, since $\Pi_{\nu}$ is a globally defined symmetric covariant tensor field of order two on $\boldsymbol{x}(M)$, we may form the contraction $\left(\Pi_{v}\right)_{G n}$ of $\Pi_{v}$ relative to the induced fundamental tensor $G_{n}$ on $x(M)$. Therefore there is exactly one normal vector field $\boldsymbol{H}$ over $\boldsymbol{x}(\boldsymbol{M})$ defined by

$$
\left\langle\boldsymbol{\nu}, d^{2} \boldsymbol{x}\right\rangle_{G_{n}}=\langle\boldsymbol{\nu}, \boldsymbol{H}\rangle,
$$

independent of the particular $\boldsymbol{\nu}$ chosen. This normal vector field $\boldsymbol{H}$ is called the mean curvature vector. It is easy to verify that

$$
\mathrm{H}=A_{i j}^{n+s} g^{i j} e_{n+s} .
$$

A submanifold is called minimal if its mean curvature vector is identically zero. 
When $m=1$ we may take $\nu$ to be the unit normal vector $e_{n+1}$ so that from equation (2.28) the second fundamental form of the hypersurface $x(M)$ at the point $x(p)$ becomes

$$
\Pi=A_{i j} \omega^{i} \omega^{j}
$$

\section{Integral formulas}

Consider two immersions $x, x^{*}$ of a $C^{2}$-Riemannian manifold $M$ of dimension $n(\geq 2)$ into a Riemannian manifold $R$ of dimension $n+m(m>0)$, and a diffeomorphism $f$ as given by the commutative diagram

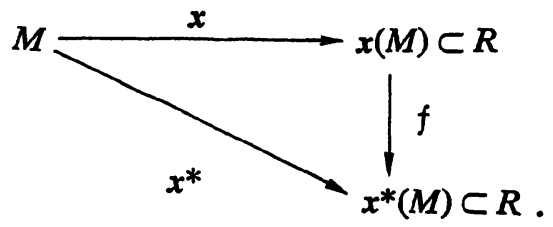

Then $\S 2$ can be applied to the submanifold $x(M)$, and for the corresponding quantities and equations for the submanifold $x^{*}(M)$ we shall use the same symbols and numbers with a star respectively. Suppose that $f$ is volumepreserving; that is, by definition it maps the volume element of one immersed submanifold into that of the other submanifold. As a consequence of this definition $f$ exists only if $M$ is oriented, and $f$ is then orientation-preserving. Now over the abstract manifold $M$ there are two induced Riemannian metrics with the same volume element, namely, $\langle d x(p), d x(p)\rangle$ and

$$
\left\langle d x^{*}(p), d x^{*}(p)\right\rangle=\langle d(f \circ x)(p), d(f \circ x)(p)\rangle .
$$

Thus the notion of frames $e_{1} \cdots e_{n}$ having measure 1 and an orientation coherent with that of $M$ has a sense in both metrics. At a point $p \in M$ any such frame can be obtained from a fixed one by a linear transformaton of determinant 1 . The volume element $\mathrm{dV}$ of the submanifold $x(M)$ is given by

$$
d V=\omega^{1} \wedge \cdots \wedge \omega^{n},
$$

where $\omega^{1} \cdots \omega^{n}$ is the coframe dual to the frame $e_{1} \cdots e_{n}$ of measure 1 . The condition for the frame $e_{1} \cdots e_{n}$ to be of measure 1 is

$$
\left\langle e_{1} \wedge \cdots \wedge e_{n}, e_{1} \wedge \cdots \wedge e_{n}\right\rangle=1,
$$

where the left-hand side is the scalar product of multivectors defined by the scalar product in the tangent space $R_{x}$ of the manifold $R$. Differentiating equation (3.2) and using the second equation of (2.22), we obtain, in consequence of $\left\langle\boldsymbol{e}_{i}, \boldsymbol{e}_{n+s}\right\rangle=0$, 


$$
\operatorname{Tr} \Omega \equiv \sum_{i} \omega_{i}^{i}=0
$$

For an ( $m \times n)$-matrix $a=\left\|a_{i j}\right\|$ and an $(n \times p)$-matrix $b=\left\|b_{j k}\right\|$, whose entries are vectors in the tangent space $R_{x}$, we shall use the notation $\langle a, b\rangle$ to denote the matrix of real numbers given by

$$
\langle\boldsymbol{a}, \boldsymbol{b}\rangle=\left\|\sum_{j}\left\langle\boldsymbol{a}_{i j}, \boldsymbol{b}_{j k}\right\rangle\right\| \text {. }
$$

In order to derive a new integral formula for two compact submanifolds $\boldsymbol{x}(M), x^{*}(M)$ under a volume-preserving diffeomorphism $f$, we shall assume that on the manifold $R$ there is a vector field $\xi$. Put

$$
<\xi, e_{n+s}>=y_{n+s},
$$

and introduce the matrix

$$
h=\left\|y_{n+1}, \cdots, y_{n+m}\right\|=\left\langle\xi,{ }^{t} a\right\rangle .
$$

By using equations (2.23), (2.26) we then have the $(1 \times n)$-matrix

$$
h^{t} \theta=\left\|y_{n+s} A_{i j}^{n+s} \omega^{j}\right\| \text {. }
$$

On the other hand, consider the orthogonal projection $\xi^{\perp}(x(p))$ of the vector $\boldsymbol{\xi}(\boldsymbol{x}(p))$ in the normal space of the submanifold $\boldsymbol{x}(\boldsymbol{M})$ at $\boldsymbol{x}(p)$, so that we have

$$
\xi^{\perp}=y_{n+s} e_{n+s} \text {. }
$$

Thus substitution of equation (3.8) in equation (2.28) gives the quadratic differential form

$$
\Pi_{\xi} \perp=\left\langle\xi^{\perp}, d^{2} x>=y_{n+s} A_{i j}^{n+s} \omega^{i} \omega^{j} .\right.
$$

In order to construct some exterior differential forms globally defined over the submanifolds $x(M), x^{*}(M)$, we further introduce the following matrices:

$$
\begin{gathered}
G=\left\langle e,{ }^{t} e\right\rangle={ }^{t} G=\left\|g_{i k}\right\|, \\
G^{*}=\left\langle f_{*}(e), f_{*}\left({ }^{t} e\right)\right\rangle={ }^{t} G^{*}=\left\|g_{i k}^{*}\right\|, \\
\Lambda=\left\|\omega_{1}, \cdots, \omega_{n}\right\|=\omega G, \\
\Lambda^{*}=\left\|\omega_{1}^{*}, \cdots, \omega_{n}^{*}\right\|=\omega G^{*}, \\
u=\Lambda e, u^{*}=\Lambda^{*} e, \\
Y=\left\langle\xi,{ }^{t} e>=\left\|y_{1}, \cdots, y_{n}\right\|,\right. \\
r=Y e,
\end{gathered}
$$




$$
\begin{gathered}
\Phi=r u^{* n-1}=\phi e_{1} \wedge \cdots \wedge e_{n}, \\
\theta=\left\|\omega^{i} \nabla_{i} \xi_{1}, \cdots, \omega^{i} \nabla_{i} \xi_{n}\right\| .
\end{gathered}
$$

The diffeomorphism $f$ is an isometry if and only if $G=G^{*}$.

Since $\phi$ is an exterior differential form of degree $n-1$ globally defined over $M$, for a compact $M$ Stokes' theorem gives

$$
\int_{M} d \phi=0 \text {. }
$$

Making use of equations (2.12), (3.18), (3.10), (2.22), (3.15), (3.16) we obtain

$$
d Y=\left\langle d \xi,{ }^{i} \boldsymbol{e}\right\rangle+\left\langle\boldsymbol{\xi}, d^{\imath} \boldsymbol{e}\right\rangle=\theta+Y^{t} \Omega+h^{\imath} \theta,
$$

from which, together with (3.16), it follows that

$$
d \boldsymbol{r}=\left[\theta+Y\left({ }^{t} \Omega+\Omega\right)+h^{2} \theta\right] \boldsymbol{e}+Y \theta a .
$$

By equations (3.11), (2.22)*, (3.13), (2.24) we have

$$
\begin{aligned}
d G^{*} & =\left\langle d f_{*}(e), f_{*}\left({ }^{t} e\right)\right\rangle+\left\langle f_{*}(e), d f_{*}\left({ }^{t} e\right)\right\rangle \\
& =\Omega^{*} G^{*}+G^{* t} \Omega^{*} \\
d \Lambda^{*} & =\left(d \omega^{*}\right) G^{*}-\omega d G^{*}=-\Lambda^{* t} \Omega^{*},
\end{aligned}
$$

so that

$$
d u^{*}=-\Lambda^{*}\left({ }^{t} \Omega^{*}+\Omega\right) e-\Lambda^{*} \theta a .
$$

By using equations (3.17), (3.21), (3.24) and the relation

$$
d\left(u^{* n-1}\right)=(n-1)\left(d u^{*}\right) u^{* n-2},
$$

we obtain

$$
\begin{aligned}
d \Phi=[\theta & \left.+Y\left({ }^{t} \Omega+\Omega\right)+h^{t} \theta\right] e u^{* n-1}-(n-1) r \Lambda^{*}\left({ }^{t} \Omega^{*}+\Omega\right) e u^{* n-2} \\
& +Y \theta a u^{* n-1}-(n-1) r \Lambda^{*} \theta a u^{* n-2}
\end{aligned}
$$

On the other hand, from the second expression of equation (3.17) we have

$$
d \Phi=(d \phi) e_{1} \wedge \cdots \wedge e_{n}+(-1)^{n-1} \phi d\left(e_{1} \wedge \cdots \wedge e_{n}\right) .
$$

Since the frames $e_{1} \cdots e_{n}$ are of measure $1, d\left(e_{1} \wedge \cdots \wedge e_{n}\right)$ contains no terms in $e_{1} \wedge \cdots \wedge e_{n}$ in consequence of (3.3). Comparison of equation (3.26) with equaton (3.27) gives 


$$
\begin{aligned}
(d \phi) e_{1} & \wedge \cdots \wedge e_{n}=\theta e u^{* n-1}+h^{t} \theta e u^{* n-1} \\
& +Y\left[\left({ }^{t} \Omega+\Omega\right) e u^{*}-(n-1) e \Lambda^{*}\left({ }^{t} \Omega^{*}+\Omega\right) e\right] u^{* n-2}
\end{aligned}
$$

By putting $w=u^{*}, \xi=\Lambda^{*}, \eta={ }^{t} \Omega^{*}$ and $\eta=\Omega$ in equation (1.8) in turn we can reduce equation $(3.28)$ to

$$
(d \phi) e_{1} \wedge \cdots \wedge e_{n}=\left[\theta+h^{t} \theta+Y\left({ }^{t} \Omega-{ }^{t} \Omega *\right)\right] e u^{* n-1} .
$$

Thus application of the integral formula (3.19) yields that for a pair of compact immersed submanifolds $\boldsymbol{x}(M), x^{*}(M)$ under a volume-preserving diffeomorphism $f$ in a Riemannian manifold $R$, on which there is a vector field $\xi$,

$$
\begin{aligned}
& \int_{M}\left\{\text { the coefficient of } e_{1} \wedge \cdots \wedge e_{n}\right. \text { in } \\
& \left.\qquad\left[\theta+h^{t} \theta+Y\left({ }^{t} \Omega-{ }^{t} \Omega^{*}\right)\right] e u^{* n-1}\right\}=0 .
\end{aligned}
$$

By the same method as above we can also derive an integral formula for two compact immersed hypersurfaces $x(M), x^{*}(M)$ in the above Riemannian manifold $R$ under a volume-preserving diffeomorphism $f$. For this purpose we shall assume $m=1$ in the remainder of this section, and introduce the following additional matrices:

$$
\begin{gathered}
v={ }^{t} \theta e, \quad v^{*}={ }^{t} \theta^{*} e, \\
\Psi=r v^{* n-1}=\psi e_{1} \wedge \cdots \wedge e_{n} .
\end{gathered}
$$

As before, for a compact manifold $M$ Stokes' theorem gives

$$
\int_{M} d \psi=0
$$

On the other hand, from the second equation of (2.22) we have

$$
d \theta=\Omega \wedge \theta,
$$

so that by means of equations (3.31), (2.22), (3.34)* a calculation similar to the one above for $d u^{*}$ yields

$$
d v^{*}=-{ }^{t} \theta^{*}\left({ }^{t} \Omega^{*}+\Omega\right) e-{ }^{t} \theta^{*} \theta e_{n+1} .
$$

Using equations (3.20), (3.21) and the relation (3.25) for $v^{*}$, from equation (3.32) we obtain

$$
\begin{aligned}
d \Psi= & {\left[\theta+Y\left({ }^{t} \Omega+\Omega\right)+y_{n+1}{ }^{t} \theta\right] e v^{* n-1}-(n-1) r^{t} \theta^{*}\left({ }^{t} \Omega^{*}+\Omega\right) e v^{* n-2} } \\
& \left.+Y \theta e_{n+1} v^{* n-1}-(n-1) r{ }^{t} \theta^{*} \wedge \theta\right) e_{n+1} v^{* n-2}
\end{aligned}
$$


Since $d\left(e_{1} \wedge \cdots \wedge e_{n}\right)$ contains no terms in $e_{1} \wedge \cdots \wedge e_{n}$ in consequence of equation (3.3), equating the terms in $e_{1} \wedge \cdots \wedge e_{n}$ on both sides of equation (3.36) we thus have

$$
\begin{aligned}
& (d \psi) e_{1} \wedge \cdots \wedge e_{n}=\left(\theta+y_{n+1}{ }^{t} \theta\right) e v^{* n-1} \\
& \quad+Y\left[\left({ }^{t} \Omega+\Omega\right) e v^{*}-(n-1) e^{t} \theta^{*}\left({ }^{t} \Omega^{*}+\Omega\right) e\right] v^{* n-2} .
\end{aligned}
$$

By putting $w=v^{*}, \xi={ }^{t} \theta^{*}, \eta={ }^{t} \Omega^{*}$ aud $\eta=\Omega$ in equation (1.8) in turn we can reduce equation (3.37) to

$$
(d \phi) e_{1} \wedge \cdots \wedge e_{n}=\left[\theta+y_{n+1}{ }^{t} \theta+Y\left({ }^{t} \Omega-{ }^{t} \Omega^{*}\right)\right] e v^{* n-1} .
$$

Thus application of the integral formula (3.33) yields that for a pair of compact immersed hypersurfaces $x(M), x^{*}(M)$ under a volume-preserving diffeomorphism $f$ in a Riemannian manifold $R$, on which there is a vector field $\xi$,

$$
\begin{aligned}
& \int_{M}\left\{\text { the coefficient of } e_{1} \wedge \cdots \wedge e_{n}\right. \text { in } \\
& \left.\qquad\left[\theta+y_{n+1}{ }^{t} \theta+Y\left({ }^{t} \Omega-{ }^{t} \Omega^{*}\right)\right] e v^{* n-1}\right\}=0 .
\end{aligned}
$$

\section{Theorems}

Let $M$ and $M^{*}$ be two $n$-dimensional $(n \geq 2) C^{2}$-Riemannian manifolds with fundamental tensors $G$ and $G^{*}$ respectively, and $f: M \rightarrow M^{*}$ a $C^{2}$-mapping. Then on $M$ there are two connexions; the Levi-Civita connexion defined by its Riemannian metric and the connexion induced by the mapping $f$ from the Levi-Civita connexion of $M^{*}$. The difference of these two connexions is a tensor field $\Delta$ of contravariant order 1 and covariant order 2 , and the construction in $\S 1$ gives a vector field $\Delta_{G} . f$ is called an isometry if $G^{*}=G$, and an almost isometry relative to $G$ if $\Delta_{G}=0$. It is clear that an isometry is also an almost isometry, since in this case $\Delta=0$.

Theorem 4.1. Let $M$ be a differentiable manifold of dimension $n \geq 2$, $x, x^{*}: M \rightarrow R$ two immersed compact submanifolds, with fundamental tensors $G, G^{*}$ respectively ${ }^{1}$, in a Riemannian manifold $R$, which admits an enlarging infinitesimal nonisometric conformal transformation $\xi$, and $f: x(M) \rightarrow x^{*}(M)$ a volume-preserving diffeomorphism. Then $f$ is an isometry, if it is an almost isometry relative to $G^{*}$, that is,

$$
\Delta_{G^{*}}=0,
$$

and the following inequality holds at every $p \in M$ :

\footnotetext{
${ }^{1}$ It should be remarked that in $\$ 3$ we have also used $G, G *$ to denote the matrices of these two tensors.
} 


$$
Q_{G} \leq Q_{G^{*}},
$$

where $Q$ is the coefficient tensor of the quadratic form $\left\langle\xi^{\perp}(x(p)), d^{2} x(p)\right\rangle$.

In particular, when $R$ is a Euclidean space $E$, this theorem is due to Chern and Hsiung [1], as in this case, in $E$ the position vector $x$ of a point in a Cartesian coordinate system with a fixed point as the origin 0 generates an enlarging infinitesimal homothetic transformation, for which the function $\rho$ in equation (2.19) is equal to 1.

Proof. In order to prove this theorem we must find $d \phi$, which is the coefficient of $e_{1} \wedge \cdots \wedge e_{n}$ on the right side of equation (3.29). For this purpose we observe that each term on the right side of equation (3.29) is of the same type as the form $\pi e u^{* n-1}$, where

$$
\pi=\left\|\pi_{1}, \cdots, \pi_{n}\right\|
$$

is a one-rowed matrix of linear differential forms on $M$. By using equations (2.21), (3.14), (3.13) we have

$$
\begin{aligned}
\pi e u^{* n-1} & =\left(\sum_{i} \pi_{i} e_{i}\right)\left(\sum_{j} \omega_{j}^{*} e_{j}\right)^{n-1} \\
& =\left(\sum \varepsilon_{i_{1}} \cdots i_{n} \pi_{i_{1}} \wedge \omega_{i_{2}}^{*} \wedge \cdots \wedge \omega_{i_{n}}^{*}\right) e_{1} \wedge \cdots \wedge e_{n},
\end{aligned}
$$

where $\varepsilon_{i_{1}} \cdots_{i_{n}}$ is equal to +1 or -1 according as $i_{1}, \cdots, i_{n}$ form an even or odd permutation of $1, \cdots, n$, and is equal to 0 otherwise, and the summation is extended over all $i_{1}, \cdots, i_{n}$ from 1 to $n$. Assuming

$$
\begin{gathered}
\pi_{i}=h_{i j} \omega^{j}, \\
H=\left\|h_{i j}\right\|,
\end{gathered}
$$

recalling $\omega_{i}^{*}=g_{i j}^{*} \omega^{j},\left|g_{i j}^{*}\right|=1$, and using elementary properties of determinants and equations (3.1), (1.2), (1.3) we can obtain

$$
\pi e u^{* n-1}=n ! H_{G^{*}}\left(e_{1} \wedge \cdots \wedge e_{n}\right) d V .
$$

On the other hand, from equations (1.2), (1.3) we have, in consequence of equation (2.19) for the submanifold $x(M)$,

$$
\begin{aligned}
\left(\nabla_{i} \xi_{j}\right)_{G^{*}} & =g^{* i j} \nabla_{i} \xi_{j} / n \\
& =\frac{1}{2 n}\left(\nabla_{i} \xi_{j}+\nabla_{j} \xi_{i}\right) g^{* i j}=\rho G_{G^{*}},
\end{aligned}
$$

where $\rho>0$. Thus substitution of $\pi=\theta$ and $\pi=h^{t} \theta$ in equation (4.7) in turn and use of equations (3.18), (4.8), (3.7), (3.9) give immediately

$$
\theta e u^{* n-1}=n ! \rho G_{G^{*}}\left(e_{1} \wedge \cdots \wedge e_{n}\right) d V,
$$




$$
h^{t} \theta e u^{* n-1}=n ! Q_{G^{*}}\left(e_{1} \wedge \cdots \wedge e_{n}\right) d V .
$$

Since $\omega=\omega^{*}$, from the first equations of (2.24), (2.24)* we have

$$
\omega \wedge\left(\Omega-\Omega^{*}\right)=0,
$$

so that we can write

$$
\omega_{i}^{k}-\omega_{i}^{* k}=a_{i j}^{k} \omega^{j}
$$

Equations (4.11), (4.12) imply

$$
a_{i j}^{k} \omega^{i} \wedge \omega^{j}=0,
$$

which gives the symmetry of $a_{i j}^{k}$ in the subscripts $i, j$, that is,

$$
a_{i j}^{k}=a_{j i}^{k} .
$$

From the properties of the forms $\Omega, \Omega^{*}$ and the definition of the tensor $\Delta$ defined at the beginning of this section it follows that the components of $\Delta$ are $a_{i j}^{k}$, so that we can write

$$
\Delta^{k}=\left\|a_{i j}^{k}\right\| \text {. }
$$

A use of equations (2.23), (2.23)*, (3.15), (4.12) yields readily the matrix

$$
Y\left({ }^{t} \Omega-{ }^{t} \Omega^{*}\right)=\left\|y_{k} a_{1 j}^{k} \omega^{j}, \cdots, y_{k} a_{n j}^{k} \omega^{j}\right\| .
$$

By putting $H=y_{k} \Delta^{k}$ in equation (4.7) we obtain

$$
Y\left({ }^{t} \Omega-{ }^{t} \Omega^{*}\right) e u^{* n-1}=n ! y_{k} \Delta_{G^{*}}\left(e_{1} \wedge \cdots \wedge e_{n}\right) d V=0,
$$

since $\Delta_{G^{*}}^{k}=0$ due to condition (4.1).

Thus equations (4.9), (4.10), (4.17) reduce the integral formula (3.30) to

$$
\int_{M}\left(\rho G_{G^{*}}+Q_{G^{*}}\right) d V=0
$$

In particular, when the two submanifolds are identical, by definition $G_{G^{*}}=1$ and the formula (4.18) becomes

$$
\int_{M}\left(\rho+Q_{G}\right) d V=0 .
$$

Subtracting equation (4.19) from equation (4.18) we obtain

$$
\int\left[\rho\left(G_{G^{*}}-1\right)+\left(Q_{G^{*}}-Q_{G}\right)\right] d V=0 \text {. }
$$


Since $\rho>0$ by the condition of the enlarging infinitesimal conformal transformation $\xi$, and $G_{G^{*}} \geq 1$ by Gårding's inequality (1.5), from the condition (4.2) of our theorem it follows that the integrand in (4.20) is nonnegative, and therefore that

$$
G_{G^{*}}=1 .
$$

By applying Lemma 1.1 of Gårding again, equation (4.21) holds only when $G=c G^{*}$ for a certain $c$. Since $G$ and $G^{*}$ are positive definite with $|G|=$ $\left|G^{*}\right|=1$, we must have $c=1$ and therefore $G=G^{*}$, which implies that $f$ is an isometry.

Formula (4.19) gives

Corollary 4.1. Suppose that a Riemannian manifold $R$ admits an enlarging infinitesimal nonisometric conformal transformation. Then any complete minimal submanifold of $R$ is noncompact.

In particular, when $R$ is a Euclidean space, this corollary is due to Chern and Hsiung [1].

By the same method as above we can have

Theorem 4.2. Let $M$ be a differentiable manifold of dimension $n \geq 2$, $x, x^{*}: M \rightarrow R$ two immersed compact hypersurfaces with fundamental tensors $G, G^{*}$ and positive definite second fundamental forms $\Pi, \Pi^{*}$, whose coefficient tensors are denoted by $A, A^{*}$ respectively, in a Riemannian manifold $R$ which admits an enlarging infinitesimal nonisometric conformal transformation $\xi$, and $f: x(M) \rightarrow x^{*}(M)$ a volume-preserving diffeomorphism. Then $\Pi=\Pi^{*}$, if $f$ is an almost isometry relative to $A^{*}$, that is,

$$
\Delta_{A^{*}}=0,
$$

and the following conditions are satisfied:

$$
\begin{gathered}
|A|=\left|A^{*}\right|, \\
G_{A^{*}} \geq G_{A}, \\
y_{n+1} \equiv\left\langle\xi^{\perp}, e_{n+1}\right\rangle>0,
\end{gathered}
$$

where $\xi^{\perp}$ is the orthogonal projection of the vector $\xi$ onto the direction along the unit normal vector $e_{n+1}$ of $x(M)$ at the point $x(p)$.

In particular, when $R$ is a Euclidean space, this theorem is due to Hsiung [3].

Proof. As in the proof of Theorem 4.1, we observe that each term on the right side of equation (3.38) is of the same type as the form $\pi e v^{* n-1}$, where $\pi$ is defined by equation (4.3). By equations (2.21), (3.31), (2.23)* we have

$$
\begin{aligned}
\pi e v^{* n-1} & =\left(\sum_{i} \pi_{i} e_{i}\right)\left(\sum_{j} \omega_{j}^{* n+1} e_{j}\right)^{n-1} \\
& =\left(\sum \varepsilon_{i_{1}} \cdots i_{n} \pi_{i_{1}} \wedge \omega_{i_{2}}^{* n+1} \wedge \cdots \wedge \omega_{i_{n}}^{* n+1}\right) e_{1} \wedge \cdots \wedge e_{n} .
\end{aligned}
$$


Making use of equations (4.5), (4.6), (2.26)*, (2.30)*, (3.1), (1.2), (1.3) and elementary properties of determinants, from equation (4.26) we can obtain

$$
\pi e v^{* n-1}=n ! H_{A^{*}}\left|A^{*}\right|\left(e_{1} \wedge \cdots \wedge e_{n}\right) d V .
$$

Thus substitution of $\pi=\theta$ and $\pi=y_{n+1}{ }^{t} \theta$ in equation (4.27) in turn and use of equations (3.18), (3.7), (3.9), (2.30) and the equation obtained from equation (4.8) by replacing $G^{*}$ by $A^{*}$ give immediately

$$
\begin{gathered}
\theta e v^{* n-1}=n ! \rho G_{A^{*}}\left|A^{*}\right|\left(e_{1} \wedge \cdots \wedge e_{n}\right) d V, \\
y_{n+1}{ }^{t} \theta e v^{* n-1}=n ! y_{n+1} A_{A^{*}}\left|A^{*}\right|\left(e_{1} \wedge \cdots \wedge e_{n}\right) d V .
\end{gathered}
$$

On the other hand, by the same argument as that used in deriving equation (4.17) we have

$$
Y\left({ }^{t} \Omega-{ }^{t} \Omega^{*}\right) e v^{* n-1}=n ! y_{k} \Delta_{\Delta^{*}}^{k}\left|A^{*}\right|\left(e_{1} \wedge \cdots \wedge e_{n}\right) d V=0,
$$

since $\Delta_{A^{*}}^{k}=0$ due to condition (4.22). Thus equations (4.28), (4.29), (4.30) reduce the integral formula (3.39) to

$$
\int_{\mathcal{M}}\left(\rho G_{A^{*}}+y_{n+1} A_{A^{*}}\right)\left|A^{*}\right| d V=0 .
$$

In particular, when the two hypersurfaces $x(M), x^{*}(M)$ are identical, $A_{A^{*}}=1$ and the formula (4.31) becomes

$$
\int_{\mathbb{A}}\left(\rho G_{A}+y_{n+1}\right)|A| d V=0 .
$$

Subtracting equation (4.32) from equation (4.31) and using condition (4.23) we obtain

$$
\int_{\mathcal{M}}\left[\rho\left(G_{A^{*}}-G_{A}\right)+y_{n+1}\left(A_{A^{*}}-1\right)\right]|A| d V=0 .
$$

Since $\rho>0$, and $A_{A^{*}} \geq 1$ by Gårding's inequality (1.5), from the conditions (4.24), (4.25) of our theorem it follows that the integrand in (4.33) is nonnegative, and therefore that

$$
A_{A^{*}}=1
$$

By applying Lemma 1.1 of Gårding again, equation (4.34) holds only when $A=c A^{*}$ for a certain c. Since by assumption $A$ and $A^{*}$ are positive definite with equal determinants, we must have $c=1$ and therefore $A=A^{*}$, which implies that $\Pi=\Pi^{*}$. 


\section{Bibliography}

[1] S. S. Chern \& C. C. Hsiung, On the isometry of compact submanifolds in Euclidean space, Math. Ann. 149 (1963) 278-285.

[2] L. Gårding, An inequality for hyperbolic polynomials, J. Math. Mech. 8 (1959) 957965.

[3] C. C. Hsiung, On the congruence of hypersurfaces, Atti Accad. Naz. Lincei Rend. Cl. Sci. Fis. Mat. Natur. (8) 37 (1964) 258-266.

[4] B. H. Rhodes, On some inequalities of Gårding, Acad. Roy. Belg. Bull. Cl. Sci. (5) 52 (1966) 594-599. 